\title{
Improving Report Generation and Delivery System of Microbiological Investigations at MRI - Sri Lanka with Concern to Turn-Around-Time, An Intervention Study
}

\author{
https://doi.org/10.3991/ijoe.v16i09.13799 \\ Pamila Adikari ( $₫)$ \\ Fiona Stanley Hospital, Perth, Australia \\ m.19604@pgim.cmb.ac.lk, Pamila.Adikari@health.wa.gov.au \\ Sunil de Alwis \\ Ministry of Healthcare, Nutrition and Indigenous Medicine, \\ Colombo, Sri Lanka \\ Lilani Karunanayake \\ Medical Research Institute, Colombo, Sri Lanka
}

\begin{abstract}
Background: Clinical Bacteriology Laboratory (CBL) of the Medical Research Institute (MRI) is a National Reference Laboratory in Sri Lanka. The service improvement research project aimed at improving the report generation and delivery system.

Methods: The mixed-method applied to study the process. Gap identification and designing of the intervention used a qualitative approach. Quantitative methods measured the effectiveness of the improvements. A package of interventions based on total quality management (TQM) principles designed with all stakeholder participation.

Results: Delay in sample transport resulted from poor communication, lack of established mechanism and weak coordination between the transport unit and wards. The paper-based report generation process was disorganized, unreliable and error-prone. Additionally, the report delivery to Hospitals revolved on the incoming ambulance or government postal service, a tied-up, poorly coordinated system. The interventions promoted coordinated communication. WHONET, a free windows-based Laboratory Information System and database software, developed by the World Health Organization for microbiology laboratories installed to generate reliable and accurate automated reports. Reports in PDF format delivered to end-users by secured email. Timeliness, accuracy, quality and uniformity of reports improved. Improvement in post-intervention turn-aroundtime was statistically significant $(\mathrm{p}<0.05)$.

Conclusion: The interventions based on TQM principles improved the process of report generation and delivery. Laboratory automation is a feasible and effective solution in the local setting. The end-user satisfaction perceived to be high on the improvements.
\end{abstract}

Keywords-Microbiology report delivery, turnaround time, WHONET, laboratory information system, Medical Research Institute of Sri Lanka. 


\section{Introduction}

The Medical Research Institute (MRI) is the foremost reference laboratory in Sri Lanka and provides microbiological services to all hospitals in the country with the highest precision and quickest turnaround times (TAT). The clinical bacteriology laboratory (CBL) of MRI equipped with state-of-the-art modern equipment to provide clinicians with accurate reports.

Sample reception and report delivery process include the collection of the sample at the ward to the delivery of the report to the same. At the ward samples are collected, details are entered in the sample register and sent to the hospital lab for transfer to MRI. At the hospital laboratory sample receipt and transfer are entered in their sample register. The samples are transported by ambulance on routine visits to Colombo. They are handed over by the ambulance helpers to the receiving counter at MRI. The Medical Laboratory Technologist (MLT) at the counter enters the details of the samples in their specimen register. CBL receives the samples in batches usually as morning/ afternoon samples and entered in the sample receipt register at CBL. A resident MLT is responsible for this during the weekends, public holidays and after working hours. Tests are performed, and results are obtained daily. Reports are generated and submitted to Consultant Clinical Microbiologist (CCM) for clinical validation, technical assessment and authorization. The finalised report is enveloped, entered in a register and sent to the specimen counter for dispatch. The reports are entered in the report receipt register at the counter. Either they are handed over to the ambulance or sent via post. The mode of issuance is noted. On receipt at the hospital laboratory, they are entered in the report receipt register. Finally, reports are entered in the 'report receipt register' at the ward. The process map is shown in figure 1.

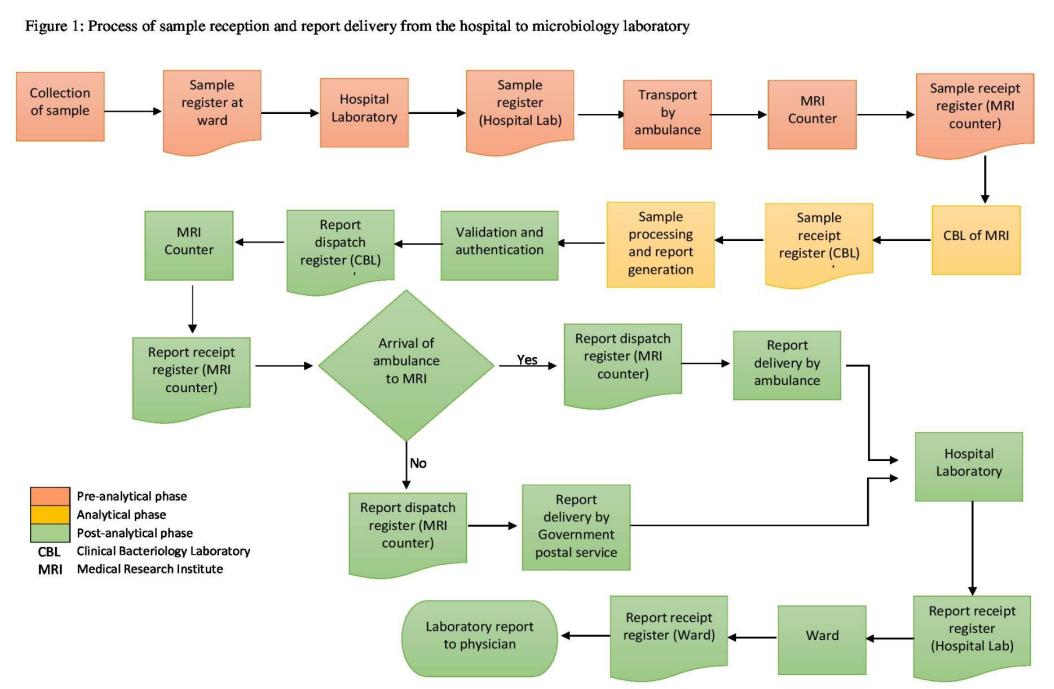

Fig. 1. Process of sample reception and report delivery from the hospital to microbiology laboratory 
The four pillars of efficient laboratory services are accuracy, precision, timeliness and authenticity. TAT is defined as the period for completing a process cycle of an investigation, commonly expressed as an average of previous such periods [1]. TAT is defined as "brain to brain TAT of physician", a cycle of events from the ordering of reports to taking action [2]. Others define TAT as the time gap between the receipt of samples to the laboratory to the availability of results [3]. TAT for an investigation is considered as a performance indicator [4]. TAT can be subdivided into three phases. Pre-analytical phase includes time taken for sample collection and transport while the analytical phase includes analysis and generation of reports. Post-analytical phase accounts for, verification of results and delivery of reports [5]. Up to $96 \%$ of contribution to TAT may be from non-analytical factors [6] while $30 \%$ of delays are pre-analytical and $20 \%$ occur at the analytical phase and $27 \%$ are caused by postanalytical problems [4].

Uncontrollable factors such as location, institutional type, bed strength and staffing levels and controllable factors such as expertise of staff, usage technology and automation, faster transport and modern report delivery techniques contribute to TAT [4]. WHONET is a windows-based automation software developed for the management and analysis of microbiological laboratory data. WHONET has been successfully used to improve quality, generate reports, detection of resistance patterns and to obtain monthly summaries in microbiology laboratories [7]. A faster TAT is a universal expectation. It will help evidence-based decision making.

Longer TAT extends patients length of stay and clinical outcome [8]. Irrational antibiotic treatment due to longer TAT can cause poor outcomes of patients and antimicrobial resistance (AMR) in microorganisms. Prolonged TAT is the commonest complaint against laboratories and leads to dissatisfaction among clinicians and patients. The level of unhappiness related to TAT of urgent samples is mostly disfavoured [4]. Prolonged TAT is a constant complaint made by specialists in hospitals especially in Special Care Baby Units and Intensive Care Units (ICU) against MRI. It contributes to ordering investigations from the private sector increasing out-of-pocket expenditure of patients. Faster TAT reduces morbidity, mortality, length of hospital stay and economic loss to the government and patient. Timely reports help clinicians to properly diagnose and decide correct treatment. This helps them select narrowspectrum antibiotics [9] minimizing development of resistant strains and iatrogenic infections [10]. Reduced length of stay results in fewer hospital-acquired infections and improved patient outcomes. Also, ill-timed reports lead to repetition of the same sample either due to accident or intentional to get a quicker report indirectly leading to an increased workload of the laboratory and cost to the hospital [11].

The aim of the study was to identify the factors that contribute to the delays, to implement interventions to improve report generation and delivery and to assess the effectiveness of these interventions. 


\section{Methodology}

CBL/MRI and Base Hospitals (BH) Kuliyapitiya and Homagama were selected as study settings. Medical, Paediatric, Premature Baby Unit, Surgical wards and the ICU were included in the study based on the frequency and number of samples. The interventional research project contained pre-and post-assessments and conducted from September 2017 to April 2018. As groundwork, several rounds of discussions were made with Medical Superintendents, Consultants, Senior MLTs, Nursing Sisters and Overseers to understand the process of management of a sample flow. Insight from these discussions helped create data collection tools. The staff at study settings were requested to maintain legible registers to help this project. Screening of MRI records showed no monthly variations of sample receipt from both hospitals during the past year. The project has 3 phases:

- Phase 1- Gap analysis

- Phase 2- Designing of the intervention

- Phase 3-Evaluation of the effectiveness of the intervention

Phase 1: Identification and assessment of gaps

Qualitative assessment: A series of key informant interviews (KII) and focus group discussions (FGD) with stakeholders were used for gap identification and gathering information for improvement of the current process. Stakeholders were the staff involved in the sample sending process at hospitals and MRI. Consultants, medical officers, ward sisters and MLTs working at the hospital laboratories were included from hospitals and Senior MLT and MLTs in the laboratory and receiving counter, Director and Deputy Director of MRI were the main stakeholders.

The KII and FGD guides were developed considering inputs from groundwork and studying internationally accepted guidelines [12], [13]. The purpose of the study was explained to the participants and written informed consent was taken. All participants were encouraged to give ideas freely. Data was collected until a saturation point was reached.

Quantitative assessment: Quantitative assessment was done using date and time information along sample flow available at MRI and hospital registers which are reliable sources of information that are supervised by the ward sisters, chief MLTs, medical officers and consultants. Random checks and active monitoring of these registers were done to ensure data quality. Following registers were utilised;

\section{Hospital:}

- Sample register at Hospital ward

- Sample Register at the hospital laboratory

- Register of receipt of investigation reports to the hospital laboratory

- Register of receipt of investigation reports to the hospital ward 
MRI:

- Sample receipt register at the counter of MRI

- Sample receipt register at clinical bacteriology laboratory

- Report dispatch book at clinical bacteriology laboratory

- Report dispatch book at MRI counter

Six time-intervals were identified and named T1 to T6. Time-intervals were measured in hours and rounded up to the nearest hour. Transport time from lab to each hospital was removed from the analysis due to the differences in distance for the two institutions.

\section{T1-T3: pre-analytical:}

- T1: Time taken for the sample to reach the hospital lab from the point of collection. Logs from sample register at ward and hospital laboratory.

- T2: Idling time at the hospital laboratory, before being transported to the MRI. Logs from a sample register at the laboratory.

- T3: Idling time at the MRI counter before reaching the CBL. Logs from specimen receipt books of MRI counter and CBL.

- T4 Analytical: gap-time between receipt of the sample at the CBL to dispatch of a printed report to the MRI counter. Logs from 'specimen receipt' and 'report dispatch registers'.

- T5/T6: Post-analytical

- T5: Time taken for a report to reach the hospital laboratory from the MRI counter. Logs from 'report dispatch book' of counter and 'report receipt book' of hospital laboratory.

- T6: Time taken for a report to reach the ward from the hospital laboratory. Logs from the 'registers of report dispatch' of the laboratory and 'report receipt' of the ward.

- Pre-testing of time-survey format, KII and FGD guides were done at BH Marawila.

\section{Phase 2: Develop interventions for improvement}

Qualitative inputs by stakeholder-suggested improvements for the lagging processes were selected based on cost, feasibility, existing resources, time for implementation and administrative feasibility. A package of interventions designed to speed the flow and that adds value to the processes were selected for implementation. Interventions were monitored by the stakeholders and minor adjustments were done to fulfil the needs of the clients.

Phase 3: Assessing the effectiveness of the improvements

The effectiveness of the interventions was analysed using qualitative and quantitative data compared with pre-interventional data.

Qualitative assessment: Measured by perceived satisfaction of stakeholders from hospitals and MRI using the similar techniques of Phase 1.

Quantitative assessment: The time survey format was used to measure the timeintervals $\mathrm{T} 1$ to $\mathrm{T} 3$. The intervention is directly emailing reports to the end users. Therefore, a T7 time-interval was measured post-intervention. 
- T7: Is the total time spent on analysis to report emailing to the end users of the hospitals. Logs were taken from 'sample receipt register' at CBL and 'sent' email $\log$.

Time values obtained were entered in the time survey format for statistical analysis. All qualitative discussions were numbered and recorded with participant's permission. Statements were purified. Common wordings were synthesized and coded into thematic areas. Each thematic area was converted to information manually. Preintervention T1, T2 and T3 time-intervals were compared with post-intervention counterparts. Average sum of pre-intervention T4, T5 and T6 time-intervals were compared with $\mathrm{T} 7$ post-intervention. Independent $\mathrm{t}$-test was used to compare the means of two groups.

Ethical clearance was obtained from the Ethics Review Committee, Medical Research Institute, Colombo on project no 37/2017. Administrative permission was obtained from Heads of the Institutes. Every step was taken to protect the confidentiality of the personally identifiable information. When designing the interventions security measures were taken to prevent access to sensitive information by an unauthorized person.

\section{Results}

Both qualitative and quantitative techniques were used to identify the gaps in sample delivery and receiving of reports. Several gaps in the pre analytical phase were revealed in the qualitative assessment. Mode of sample transport in both hospitals was the ambulance. Gaps identified, and the solutions suggested by the participants were as follows.

1. Undue delay during transport to MRI: According to the consultant paediatrician "ideally a neonate's blood should reach the MRI on the same day it was collected. However, samples tend to be delayed more than three days in hospital labs"

2. Poor coordination between the transport department (TD) and hospital labParticipants felt that the communication between the ward and the TD is poor. ICU doctors pointed out that "even though ambulances travel to Colombo on most days, the samples are left behind and not transported to MRI daily. The TD should be responsible for sending samples to the MRI. They should contact the laboratory and inform them before an ambulance leaves for Colombo" was the opinion of the participants.

3. Poor documentation- Discussions with key informants and focus group members revealed that documentation is disorganized. The registers lack basic information for tracing samples or reports. Focus group participants from the hospital laboratory suggested proper maintenance of the sample register with the information required for tracing the reports.

Gaps recorded in the analytical phase were: 
1. Outdated and error-prone report generation system: The CCM mentioned that the CBL possesses the latest technology for the analysis. However, the reports and the statistics are generated manually. "The report delivery system we use here is outdated and finalizing statistical reports at the end of the month is a time waster and burden for the staff' expressing her need for an advanced system. The reports were generated by altering previously word-processed reports. This is an error-prone mechanism as well as a time-consuming process. There is a high rate of transcriptional errors in reports.

2. Time for report generation- Also, MLT's should document all information of the sample manually in many books for monthly statistics and reagent ordering. The CCM suggested, "we should adopt WHONET software in microbiology which is very useful for generating reports and statistics".

Following were the deficiencies in the post-analytical phase:

1. Delayed validation time: Validation of paper-based reports needs the physical presence of CCM in the lab. She revealed that academic, training, teaching and administrative work prevent her from validating reports as they arrive.

2. Delayed delivery of reports: It was found that the paper-based reports which are posted do not reach the hospitals on time. Hospital staff is disappointed regarding the untimely reports. They get the microbiology reports over the telephone which may lead to erroneous interpretation. Further, contacting the MRI is difficult from 8 a.m. to 12 noon, which is the peak time with morning ward rounds.

3. The disorganized report issuance at MRI counter: Handing over of reports to the ambulances is uncoordinated. E.g. reports are not grouped according to hospitals, ambulances leave reports at the MRI counter etc.

4. Use of ICT technology for report delivery: Delays in report delivery can be minimized by using ICT techniques. Email services can be securely used to transmit reports. Mobile devices can be used to access email.

Several interventions were designed based on feasibility and cost. An internal circular was issued by the relevant hospital heads to improve coordination between units and transport. The staff was educated on the importance of maintaining legible registers. All supervisory staff including consultants, senior house officers, nursing sisters, senior MLTs were asked to monitor the registers. Research team randomly checked these registers. Introduced WHONET software by WHO, to the CBL. A workshop was organized for doctors and MLTs to familiarize the software. WHONET 2018 was installed on four computers of the CBL and suitably configured by the CCM and PI to serve multiple institutions and to be user-friendly. A backup was saved in a compact disc. The computers were networked using a wireless network. The MLTs entered the data supervised by the CCM and PI. Open-source PDF printer software was used to create PDF reports and the MLTs were trained. Separate folders were created for hospitals to prevent any mix-ups. Email based system for report validation and report delivery established. Email addresses were created for CBL, ICU, PBU and one common address for other units of BH. Reports were emailed to CCM for validation as and when a report is generated. The hospital medical superintendents were informed to 
nominate responsible persons to check emails. Consultants were given the email and passwords on laminated cards. Units with computers checked their emails and the hospital planning unit printed reports for the others.

Number of microbiology samples sent from PBU, ICU and other wards are listed below (Table 1). Most numbers of samples are sent from the PBU and ICU in both BHs. The independent t-test requires that the measured time interval which is the dependent variable is approximately normally distributed within pre and post intervention groups. Data set was explored using SPSS for normality and concluded that it comes from a normal distribution. Quantitative assessment compared post-interventions and preinterventional time intervals. A significant improvement was seen in specimen transport within the hospital $\mathrm{p}<0.05$ (Table 2 ). A statistically significant reduction was seen in idling-time of specimens at hospital lab $\mathrm{p}<0.05$ (Table 3). A significant reduction in idling-time of samples at the MRI counter was seen. $\mathrm{p}<0.05$ (Table 4). A significant reduction in time with analytical and post-analytical phases at CBL was seen (Table 5).

Perceived satisfaction of end users at hospitals and MRI were assessed qualitatively using KII and FGD. Improvements in the sample sending process resulted in coordinated sample transport. The hospital laboratory confirmed that the TD contacted them for samples before leaving for Colombo. "After the letter from MS, the ambulances informed us before leaving for Colombo. Routine transfers are informed on the previous day so that we have enough time to get down all the samples" the chief MLT of one hospital said. Improvements in documentation increased traceability of samples. Improvements after introducing WHONET software at CBL resulted in drastic reduction in errors with manual generation of reports. "Earlier we used to type one report after the other, manually entering the fields on a pre-designed template. We often made mistakes and they were revealed at validation by the CCM" the laboratory MLTs mentioned. This software can handle a large bulk of reports at once and saves time in printing. "We could group the reports according to the hospital and print at once. Therefore, no sorting of reports needed" the MLTs elaborated. Reports had fewer errors in relation to organism names and antibiotics. WHONET auto-interpret antibiotic sensitivity and reduce errors and save time. CCM can validate results at any time even weekends and public holidays by email shortening TAT. Reduced documentation work for the laboratory MLTs recorded. "We can use the time spent on generating statistics for the analytical work now and easy to get information to place orders for the next quarter. This has tremendously reduced our work burden" the SMLT mentioned. All consultants of units believed the report delivery timeliness has greatly improved after the intervention. One physician expressed "earlier it took several days to get a report. Now we get them within 3 to 4 days of sending the sample". The ward doctors felt that their work was eased as the time spent for chasing microbiology reports were minimized. This has resulted in significant time savings in the morning ward rounds. PBU medical officer mentioned "it is easy to check the mail and get the reports. This saves a lot of my time". Connectivity problems and availability of email hindered accessibility in some units in both hospital settings. Reduction in telephone communication reduced transcription errors which affect patient safety. Medical officer in ICU said, "Emailing reports minimize errors that we make when writing down results over the phone" improving patient safety ultimately. At CBL, reduction in incoming 
calls has resulted in time saving, stress reduction and better focusing of work for MLT's and other staff. Improved quality, uniformity and the completeness of all necessary fields was mentioned by consultant paediatrician. Reduction of transcription errors associated with manual copying noted by CCM.

\section{Discussion}

This is a process improvement research project to improve report generation and delivery system of CBL of MRI. Quantitative assessment on TAT and qualitative assessment on perceived satisfaction on process improvement recorded following implementation of stakeholder-led package of total quality improvement interventions.

This study used process mapping as the technique to study PDCA cycle for continual improvement of the process. These techniques have been used successfully on previous occasions [14], [15].

Secondary data has been used to assess TAT in a similar process improvement project by Holland et. al.

According to our results, the major contribution for faster TAT was from analytical and post-analytical interventions. Hawkins 2007, records that delays in all phases were equally contributing for the TAT. The differences can be attributed to paper-based report delivery systems, difficulties in handling high turnover of results due to manual generation of reports and longer laboratory post-analytical delays because of wide geographical variations.

Present study recorded shortened pre-analytical TAT by improved coordination of sample transport, a recommended strategy by CDC in a similar resource poor setting [16]. Quality of documentation and traceability of investigations could be improved by participation of stakeholders as shown by a team led by Saravi [17]. WHONET based automation and email-based validation used in this project helped to reduce errors in reporting and shortened time for validation and authorization. Electronic report generation and secured email-led reporting contributed to faster TAT. A University Hospital study recorded faster TAT by laboratory automation [18].

The present study used email-based pdf formatted report delivery which is better than SMS-based report delivery attempted by Jian and team due to the presentation, readability and security [19]. Availability of high-speed data transmission facilities in all major cities was an advantage to adopt pdf report transmission in this research project. Unfortunately, few wards lacked internet-enabled computers and the hospital planning unit printed the reports and disseminated to wards.

The perceived satisfaction of participants was high over the coordinated sample sending, improved documentation, and faster TAT as a result of ICT based automation and report transmission as recorded by Goswami and Lee-Lewandrowski [6], [20]. Automation of CBL with WHONET software greatly helped to overcome many issues in the laboratory. Inability to include all hospitals because of time and financial constraints and difficulties in generalisation of some context specific variables are limitations of the present study. 


\section{Conclusion and Recommendation}

\subsection{Conclusion}

The report generation and delivery process at CBL of MRI was inconsistent and disfavoured by the clinicians for longer TATs. Poor coordination between the TD and wards with weak documentation were the main problems identified in the pre-analytical phase. The analytical phase affected by error-prone manual report generation and the laboratory staff maintained many routine registers. The post-analytical phase was impeded by delayed validation and disorganised report delivery mechanism.

These gaps were overcome by improving the communication between the TD and wards, introducing automated report generation using free software; WHONET, developed by the World Health Organization. WHONET was useful in the generation of statistics for AMR surveillance and to speed up the processes at CBL. Reports were transmitted to the end users through secured email.

End users convinced over the improved TAT, reduction of errors, quality and completeness of reports. The staff of CBL of MRI consillated over minimal documentation, rapid generation of report and statistics.

All measured time intervals of specimen transfer, specimen idling, and report generation and delivery were markedly reduced and were statistically significant.

\subsection{Recommendations}

1. Process improvement projects based on total quality improvement principles with all stakeholder participation could be applied to improve laboratory report generation and delivery process.

2. Electronic-based transmission of laboratory reports can be recommended as a suitable method for local setting.

3. Introduce WHONET software to local microbiology laboratories after suitable configuration

4. Hospital administrators should adopt internal mechanisms to improve coordination between TD and wards to minimize transport delays.

5. A larger study involving several study settings is recommended to test the effectiveness on total report generation and delivery process.

\subsection{Suggestions for future research}

Even after intervention, idling time at the hospital and MRI counter was still high. Future intervention research projects should address the specific gaps in these areas and suggest suitable interventions. 


\section{$6 \quad$ References}

[1] H. P. Pati and G. Singh, "Turnaround Time (TAT): Difference in Concept for Laboratory and Clinician," Indian J. Hematol. Blood Transfus., vol. 30, no. 2, pp. 81-84, Jun. 2014, https://doi.org/10.1007/s12288-012-0214-3

[2] G. D. Lundberg, "Acting on Significant Laboratory Results," JAMA J. Am. Med. Assoc., vol. 245, no. 17, p. 1762, May 1981, https://doi.org/10.1001/jama.1981.03310420052033

[3] J. I. Westbrook, A. Georgiou, and M. I. Rob, "Computerised order entry systems: sustained impact on laboratory efficiency and mortality rates?" Stud. Health Technol. Inform., vol. 136, pp. 345-350, 2008.

[4] R. C. Hawkins, "Laboratory turnaround time," Clin. Biochem. Rev., vol. 28, no. 4, pp. 179194, Nov. 2007.

[5] H.-J. Chung, W. Lee, S. Chun, H.-I. Park, and W.-K. Min, "Analysis of turnaround time by subdividing three phases for outpatient chemistry specimens," Ann. Clin. Lab. Sci., vol. 39, no. 2, pp. 144-149, 2009.

[6] B. Goswami, B. Singh, R. Chawla, V. K. Gupta, and V. Mallika, "Turn Around Time (TAT) as a Benchmark of Laboratory Performance," Indian J. Clin. Biochem., vol. 25, no. 4, pp. 376-379, Oct. 2010, https://doi.org/10.1007/s12291-010-0056-4

[7] A. N. Ghosh et al., "Application of WHONET in the Antimicrobial Resistance Surveillance of Uropathogens: A First User Experience from Nepal," J. Clin. Diagn. Res. JCDR, vol. 7, no. 5, pp. 845-848, May 2013, https://doi.org/10.7860/jcdr/2013/5193.2955.

[8] L. L. Holland, L. L. Smith, and K. E. Blick, "Total Laboratory Automation Can Help Eliminate the Laboratory as a Factor in Emergency Department Length of Stay," Am. J. Clin. Pathol., vol. 125, no. 5, pp. 765-770, May 2006, https://doi.org/10.1309/3j5p9vjrup4u5ru5.

[9] J. Barenfanger, C. Drake, and G. Kacich, "Clinical and financial benefits of rapid bacterial identification and antimicrobial susceptibility testing," J. Clin. Microbiol., vol. 37, no. 5, pp. 1415-1418, May 1999. https://doi.org/10.1128/jcm.37.5.1415-1418.1999

[10] B. W. Buchan, K. M. Riebe, and N. A. Ledeboer, "Comparison of the MALDI Biotyper System Using Sepsityper Specimen Processing to Routine Microbiological Methods for Identification of Bacteria from Positive Blood Culture Bottles," J. Clin. Microbiol., vol. 50, no. 2, pp. 346-352, Feb. 2012, https://doi.org/10.1128/jcm.05021-11

[11] B. Dey, J. N. Bharti, and M. Chakraborty, "Laboratory Turnaround Time," Int. J. Health Sci. Res., vol. 3, no. 5, 2013.

[12] B. DiCicco-Bloom and B. F. Crabtree, "The qualitative research interview," Med. Educ., vol. 40, no. 4, pp. 314-321, Apr. 2006, https://doi.org/10.1111/j.1365-2929.2006.02418.x

[13] R. A. Krueger, "Designing and Conducting Focus Group Interviews." University of Minnesota, 2002.

[14] R. Damelio, The Basics of process mapping, 2nd ed. New York: CRC/Productivity Press, 2011.

[15] Ronald Moen and C. Norman, "Evolution of the PDCA cycle," 2006.

[16] Center for Disease Control and Prevention, "Guidance for Developing a Plan for Interfacility Transport of Persons Under Investigation or Confirmed Patients with Ebola Virus Disease in the United States| Emergency Services| Clinicians| Ebola (Ebola Virus Disease) | CDC," 23-May-2018. [Online]. Available: https://www.cdc.gov/vhf/ebola/clinicians/emergencyservices/interfacility-transport.html. [Accessed: 18-Jun-2018]. https://doi.org/10.1016/b9780-12-804230-4.00007-8

[17] B. Saravi et al., "Documentation of Medical Records in Hospitals of Mazandaran University of Medical Sciences in 2014: a Quantitative Study," Acta Inform. Medica, vol. 24, no. 3, p. 202, 2016, https://doi.org/10.5455/aim.2016.24.202-206 
[18] S. Angeletti et al., "Laboratory Automation and Intra-Laboratory Turnaround Time: Experience at the University Hospital Campus Bio-Medico of Rome," J. Lab. Autom., vol. 20, no. 6, pp. 652-658, Dec. 2015, https://doi.org/10.1177/2211068214566458.

[19] W.-S. Jian et al., "LabPush: A Pilot Study of Providing Remote Clinics with Laboratory Results via Short Message Service (SMS) in Swaziland, Africa," PLoS ONE, vol. 7, no. 9, p. e44462, Sep. 2012, https://doi.org/10.1371/journal.pone.0044462.

[20] E. Lee-Lewandrowski, D. Corboy, K. Lewandrowski, J. Sinclair, S. McDermot, and T. I. Benzer, "Implementation of a point-of-care satellite laboratory in the emergency department of an academic medical center. Impact on test turnaround time and patient emergency department length of stay," Arch. Pathol. Lab. Med., vol. 127, no. 4, pp. 456-460, Apr. 2003, https://doi.org/10.1097/00134384-200209000-00004

\section{Authors}

Dr Pamila Adikari, MBBS, MSc (Medical Administration), MD (Medical Administration), International Research Fellow, Fiona Stanley Hospital, Perth, Western Australia. Email: m.19604@pgim.cmb.ac.lk, Pamila.Adikari@health.wa.gov.au

Dr Sunil de Alwis, MSc, MD, Consultant Healthcare Management and Additional Secretary (Medical Services), Ministry of Healthcare Nutrition and Indeginous Medicine, Colombo, Sri Lanka

Dr (Mrs) Lilani Karunanayake, MBBS, PG Dip (Med Microbiol), MD, Consultant Clinical Microbiologist, Medical Research Institute, Colombo, Sri Lanka

Article submitted 2020-02-17. Resubmitted 2020-05-10. Final acceptance 2020-05-22. Final version published as submitted by the authors. 


\section{Appendix}

Table 1. Frequency distribution of microbiology samples

\begin{tabular}{|l|c|c|c|c|c|c|c|}
\hline & & \multicolumn{3}{|c|}{ Pre-intervention } & \multicolumn{3}{c|}{ Post-intervention } \\
\hline & $\boldsymbol{n}$ & $\boldsymbol{P B U}$ & $\boldsymbol{I C U}$ & Other & $\boldsymbol{P B U}$ & ICU & Other \\
\hline BH Kuliyapitiya & 103 & 51 & 29 & 23 & 53 & 36 & 12 \\
\hline BH Homagama & 190 & 93 & 59 & 38 & 89 & 61 & 35 \\
\hline Total & 293 & & & & & & \\
\hline
\end{tabular}

Table 2. Comparison of pre-and post-intervention time of specimen transport within hospital (T1)

\begin{tabular}{|c|c|c|c|c|}
\hline \multicolumn{2}{|c|}{ Pre-intervention T1 (hours) } & \multicolumn{2}{|c|}{ Post-intervention T1 (hours) } & p-value \\
\hline $\boldsymbol{n}$ & Mean \pm SD & $\boldsymbol{n}$ & Mean $\pm \boldsymbol{S D}$ & \\
\hline 293 & $2.88 \pm 1.38$ & 286 & $2.49 \pm 1.13$ & $<0.05$ \\
\hline
\end{tabular}

Table 3. Comparison of pre-and post-intervention for idling time at hospital laboratory (T2)

\begin{tabular}{|c|c|c|c|c|}
\hline \multicolumn{2}{|c|}{ Pre-intervention T2 (hours) } & \multicolumn{2}{|c|}{ Post-intervention T2 (hours) } & p-value \\
\hline $\boldsymbol{n}$ & Mean \pm SD & $n$ & Mean \pm SD & \\
\hline 293 & $63.40 \pm 12.17$ & 286 & $30.75 \pm 9.56$ & $<0.05$ \\
\hline
\end{tabular}

Table 4. Comparison of pre-and post-intervention for idling time at MRI counter (T3)

\begin{tabular}{|c|c|c|c|c|}
\hline \multicolumn{2}{|c|}{ Pre-intervention T3 (hours) } & \multicolumn{2}{|c|}{ Post-intervention T3 (hours) } & p-value \\
\hline $\boldsymbol{n}$ & Mean $\pm \boldsymbol{S D}$ & $\boldsymbol{n}$ & Mean \pm SD & \\
\hline 293 & $12.79 \pm 4.39$ & 286 & $9.40 \pm 2.85$ & $<0.05$ \\
\hline
\end{tabular}

Table 5. Comparison of average sum of T4, T5, T6 pre-intervention with T7 post-intervention at $\mathrm{CBL}$

\begin{tabular}{|c|c|c|l|l|}
\hline $\begin{array}{c}\text { Pre-intervention average of sum of T4, } \\
\text { T5, T6 (hours) }\end{array}$ & \multicolumn{2}{c|}{ Post-intervention T7 (hours) } & p-value \\
\hline $\boldsymbol{n}$ & Mean \pm SD & $\boldsymbol{n}$ & Mean \pm SD & \\
\hline 293 & $248.56 \pm 81.06$ & 286 & $68.68 \pm 21.98$ & $<0.05$ \\
\hline
\end{tabular}

